\title{
Approximating Planar Rotations
}

\author{
I. Sterling ${ }^{1}$ and T. Sterling ${ }^{2}$ \\ ${ }^{1}$ Department of Mathematics, University of Toledo, \\ Toledo, $\mathrm{OH} 43606-3390$, USA \\ isterlin@math.utoledo.edu \\ ${ }^{2} 1715$ Chandler, Ann Arbor, MI 48105, USA
}

\begin{abstract}
We study the problem of approximating a rotation of the plane, $\alpha: R^{2} \rightarrow R^{2}$, $\alpha(x, y)=(x \cos \theta+y \sin \theta, y \cos \theta-x \sin \theta)$, by a bijection $\beta: Z^{2} \rightarrow Z^{2}$. We show by an explicit construction that $\beta$ may be chosen so that $\sup _{z \in Z^{2}}|\alpha(z)-\beta(z)| \leq$ $(1 / \sqrt{2})\left((1+r) / \sqrt{1+r^{2}}\right)$, where $r=\tan (\theta / 2)$. The scheme is based on those invented and patented by the second author in 1994 .
\end{abstract}

\section{Introduction}

We study the problem of approximating planar rotations (about the origin)

$$
\alpha: R^{2} \rightarrow R^{2}, \quad \alpha(0,0)=(0,0),
$$

by $1-1$, onto mappings

$$
\beta: Z^{2} \rightarrow Z^{2}
$$

of the integer lattice $Z^{2}=\left\{(x, y) \in R^{2} \mid x, y \in Z\right\}$. $\beta$ is said to approximate $\alpha$ with error

$$
e(\beta, \alpha)=\sup _{z \in Z^{2}}\{|\beta(z)-\alpha(z)|\} .
$$

A scheme $S$ associates a $\beta$ to every $\alpha$. That is, if $K$ is the space of rotations and $L$ is the space of $1-1$, onto lattice maps, then

$$
S: K \rightarrow L
$$

The error of a scheme $S$ is

$$
E_{S}=\sup _{\alpha \in K}\{e(S(\alpha), \alpha)\}
$$

We seek schemes $S$ whose error $E_{S}$ is as low as possible. 
In Section 4 we define a scheme $T$ and prove

$$
E_{T} \leq \frac{\sqrt{2+\sqrt{2}}}{2} \approx 0.924 .
$$

Numerical investigations (see Section 5) indicate that this is actually an equality

$$
E_{T}=\frac{\sqrt{2+\sqrt{2}}}{2} .
$$

The estimate in (1) is a special case of our main theorem (Section 4). Our main theorem gives the error bound for rotation by an angle $\theta(0 \leq \theta \leq \pi / 4)$ for the T-Scheme to be

$$
\frac{1}{\sqrt{2}} \frac{1+r}{\sqrt{1+r^{2}}},
$$

where $r=\tan (\theta / 2)$. For some angles our T-Scheme is not optimal-this is also discussed in Section 5.

In Section 5 we prove that, for every scheme $S$,

$$
E_{S} \geq \frac{2+\sqrt{2}}{4} \approx 0.853
$$

The authors are grateful to R. Stong for the idea behind Theorem 5.5. We also study the lower bound as a function of the rotation angle.

We would also like to thank the referee for noting that the "irrational case" could be incorporated into the "rational case" at the theory level. Numerically the cases differ.

All the schemes in this paper are modifications of the schemes invented and patented by the second author in 1994 [T]. We refer the reader to [T] for an extensive discussion of the importance of rotation schemes in image processing.

\section{Notation}

Let $J_{R}=[0, \pi / 4]$ and $I_{R}=[0, \sqrt{2}-1]$. The schemes defined in this paper are defined for angles $\theta \in J_{R}$. Such angles enjoy many nice properties. In particular, if $\tan (\theta / 2)=r$, then we have $\sin \theta=2 r /\left(1+r^{2}\right)$ and $\cos \theta=\left(1-r^{2}\right) /\left(1+r^{2}\right)$. Note that $\tan (\theta / 2)$ maps $J_{R} 1-1$, onto $I_{R}$ and that, without loss of generality, it suffices to consider angles in $J_{R}$. For $r \in I_{R}$,

$$
\alpha_{r}:=\text { rotation by the angle } 2 \tan ^{-1} r .
$$

Remark 2.1. We work in pixel coordinates (in particular, $y$ increases as it goes down).

Remark 2.2. The blocks defined below do contain their left and upper edges, but not their right or lower edges (except in degenerate cases).

Remark 2.3. $[x]:=\operatorname{Floor}(x):=x-x \bmod 1$ (i.e., the greatest integer less than or equal to $x)$. Note that $x-1<[x] \leq x<[x]+1$. 

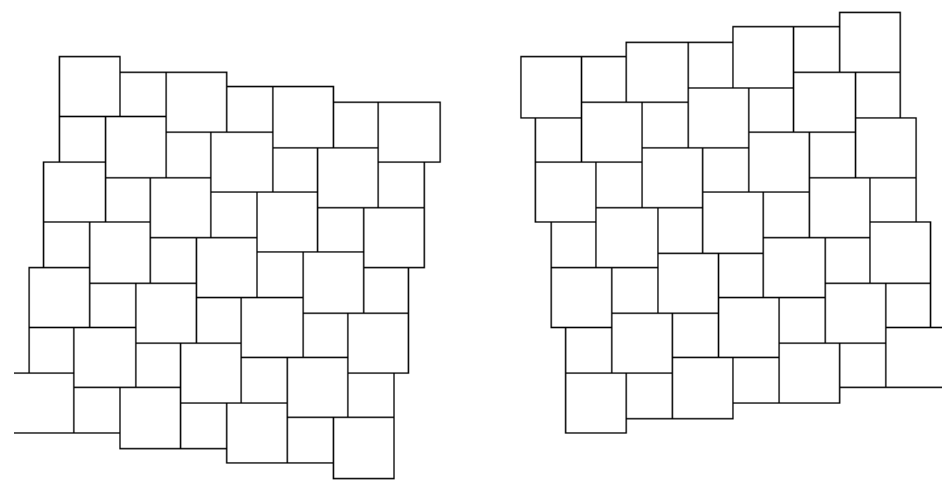

Fig. 1. $\quad P$ and $P^{\prime}$ arrays for $m / n=1 / 7$.

\section{The $P$ Array}

A block is a rectangle $B(x, y, w, h)$ in the plane with the upper left corner at $(x, y)$, with width $w$ and height $h$. We tile the plane into an array $P$ of blocks $B(x, y, w, h)$ described below. The array $P$ is transformed, block by block, to a new array $P^{\prime}$ of blocks $B^{\prime}\left(x^{\prime}, y^{\prime}, w^{\prime}, h^{\prime}\right)$.

Assume that $\tan (\theta / 2)=r \in I_{R}$. Let $R=1 / r$. The idea is that $P$ will give a tiling which is tilted by about an angle $-\theta / 2$ and $P^{\prime}$ will tilt by roughly $\theta / 2$ (see Figs. 1 and 2). The blocks in the arrays are labeled by integers $a$ and $b$. The location of any block in the array $P$ can be determined as follows: the upper left corner of any block $B(a, b)$ (where $a+b$ is even) has an $x$-coordinate $x_{\ell}(a, b)$ and a $y$-coordinate $y_{u}(a, b)$ given by

$$
\begin{aligned}
& x_{\ell}(a, b)=\left[\frac{a R-b+\zeta}{2}\right], \\
& y_{u}(a, b)=\left[\frac{a+b R+\eta}{2}\right] .
\end{aligned}
$$
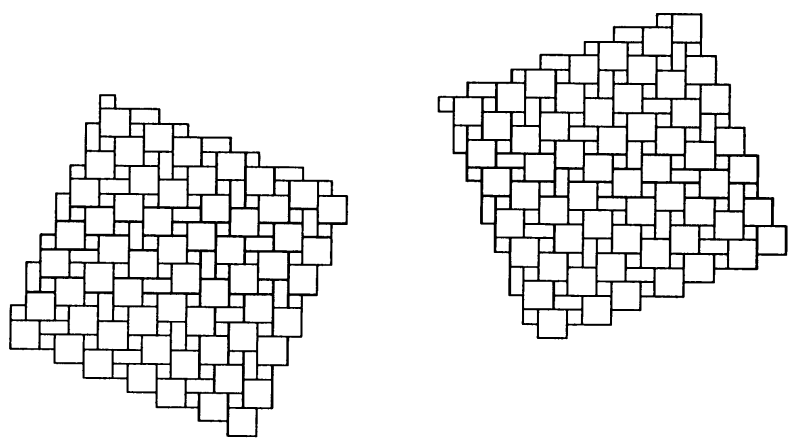

Fig. 2. $\quad P$ and $P^{\prime}$ arrays for $m / n=2 / 5$. 
The choice of $\zeta$ and $\eta$ is central to optimizing the scheme (see Section 5). In [T] the choice was $\zeta=0$ and $\eta=1 / m$ (whenever $r=m / n$ ). For our T-Scheme we choose

$$
\zeta=\frac{3-R}{2}, \quad \eta=\frac{3-R}{2}
$$

The size of the block $B(a, b)$ is defined by determining the location of the lower and right sides. The lower and right sides have the same $x$ - and $y$-coordinates as the adjoining sides of the contiguous blocks. The $x$-coordinate of the right side $x_{r}(a, b)$ is

$$
x_{r}(a, b)=x_{\ell}(a+1, b-1)=\left[\frac{a R-b+R+1+\zeta}{2}\right]
$$

and the $y$-coordinate of the lower side $y_{\ell}(a, b)$ is

$$
y_{\ell}(a, b)=y_{u}(a+1, b+1)=\left[\frac{a+b R+1+R+\eta}{2}\right] .
$$

The bounds of the blocks having $a+b$ odd are determined from the four blocks that surround it according to the following:

$$
\begin{aligned}
& x_{\ell}(a, b)=x_{r}(a-1, b)=\left[\frac{a R-b+1+\zeta}{2}\right], \\
& y_{u}(a, b)=y_{\ell}(a, b-1)=\left[\frac{a+b R+1+\eta}{2}\right], \\
& x_{r}(a, b)=x_{\ell}(a+1, b)=\left[\frac{a R-b+R+\zeta}{2}\right], \\
& y_{\ell}(a, b)=y_{u}(a, b+1)=\left[\frac{a+b R+R+\eta}{2}\right] .
\end{aligned}
$$

Finally we have

$$
\begin{aligned}
w & =x_{r}-x_{\ell}, \\
h & =y_{\ell}-y_{u} .
\end{aligned}
$$

Lemma 3.1. Every point in $R^{2}$ is in at most one block.

Proof. This is a result of the following implications.

$$
\begin{aligned}
& A \geq a+1, B \leq b \quad \Rightarrow \quad x_{r}(a, b) \leq x_{\ell}(A, B), \\
& A \geq a, \quad B \geq b+1 \quad \Rightarrow \quad y_{\ell}(a, b) \leq y_{u}(A, B), \\
& A \leq a-1, B \geq b \quad \Rightarrow \quad x_{r}(A, B) \leq x_{\ell}(a, b), \\
& A \leq a, B \leq b-1 \quad \Rightarrow \quad y_{\ell}(A, B) \leq y_{u}(a, b) .
\end{aligned}
$$

Lemma 3.2. Every point in $R^{2}$ is in some block. 
Proof. Let

$$
\operatorname{Row}(\bar{b}):=\bigcup_{-\infty<a<\infty} B(a, \bar{b}) .
$$

$x_{r}(a, \bar{b})=x_{\ell}(a+1, \bar{b})$ implies that, for all $x \in R$,

$$
x_{\ell}(a, \bar{b}) \leq x<x_{r}(a, \bar{b})
$$

for some unique $a \in Z$.

Define $(x, y)$ to be "below" (resp. "above") $\operatorname{Row}(\bar{b})$ if $y \geq y_{\ell}(a, \bar{b})$ (resp. $y<$ $y_{u}(a, \bar{b})$ ), where $a$ satisfies 2 . Note that every point in $\operatorname{Row}(\bar{b})$ is "above" every point in $\operatorname{Row}(\bar{b}+1)$. Thus, if $(x, y)$ is in no block, there must exist a $\bar{b}$ with $(x, y)$ "below" $\operatorname{Row}(\bar{b})$ and "above" $\operatorname{Row}(\bar{b}+1)$. This means that for some $a$ and $a^{\prime}$ we have

$$
x_{\ell}(a, \bar{b}) \leq x<x_{r}(a, \bar{b}), \quad y \geq y_{\ell}(a, \bar{b}),
$$

and

$$
x_{\ell}\left(a^{\prime}, \bar{b}+1\right) \leq x<x_{r}\left(a^{\prime}, \bar{b}+1\right), \quad y<y_{\ell}\left(a^{\prime}, \bar{b}+1\right) .
$$

Now assume $a+b$ is even. Since $A \leq a-1$ implies $x_{r}(A, \bar{b}+1) \leq x_{\ell}(a, \bar{b})$ and $A \geq a+2$ implies $x_{\ell}(A, \bar{b}+1) \geq x_{r}(a, \bar{b})$ we see that either $a^{\prime}=a$ or $a^{\prime}=a+1$. However, $y_{u}(a, \bar{b}+1)=y_{\ell}(a, \bar{b})$ and $y_{u}(a+1, \bar{b}+1)=y_{\ell}(a, \bar{b})$. In both cases this is a contradiction. The case $a+b$ odd is similar.

\section{The T-Scheme}

Having calculated the position and size of the blocks $B(x, y, w, h)$, the next step is to calculate the position to which the blocks are to be moved. The blocks in the new array are specified by $B^{\prime}\left(x^{\prime}, y^{\prime}, w^{\prime}, h^{\prime}\right)$.

$$
\begin{gathered}
x^{\prime}= \begin{cases}{\left[\left(a R+b+\zeta^{\prime}\right) / 2\right]} & \text { if } a+b \text { is even, } \\
{\left[\left(a R+b+1+\zeta^{\prime}\right) / 2\right]} & \text { if } a+b \text { is odd, }\end{cases} \\
y^{\prime}= \begin{cases}{\left[\left(-a+b R+\eta^{\prime}\right) / 2\right]} & \text { if } a+b \text { is even, } \\
{\left[\left(-a+b R+1+\eta^{\prime}\right) / 2\right]} & \text { if } a+b \text { is odd, }\end{cases} \\
w^{\prime}=w, \quad h^{\prime}=h .
\end{gathered}
$$

For our T-Scheme we choose $\zeta^{\prime}=\zeta$ and $\eta^{\prime}=\eta$.

Given $r \in I_{R}$ the transformation of $P$ into $P^{\prime}$ defines a map $T\left(\alpha_{r}\right)$ as follows: by Lemmas 3.1 and 3.2 we have that every point $z=(x, y) \in Z^{2}$ is (uniquely) of the form

$$
\begin{aligned}
& x=x_{\ell}(a, b)+u, \\
& y=y_{u}(a, b)+v,
\end{aligned}
$$

with $0 \leq u<w(a, b)$ and $0 \leq v<h(a, b)$.

$$
T\left(\alpha_{r}\right)(x, y):=\left(x^{\prime}, y^{\prime}\right)
$$


where

$$
\begin{aligned}
& x^{\prime}=x_{\ell}^{\prime}(a, b)+u, \\
& y^{\prime}=y_{u}^{\prime}(a, b)+v .
\end{aligned}
$$

Lemma 4.1. For all $r \in I_{R}, T\left(\alpha_{r}\right)$ is $1-1$.

Proof. We compute that

$$
\begin{aligned}
x_{r}^{\prime}(a, b) & = \begin{cases}x_{\ell}^{\prime}(a+1, b+1)=[(a R+b+R+1+\zeta) / 2] & \text { if } a+b \text { is even, } \\
x_{\ell}^{\prime}(a+1, b)=[(a R+b+R+\zeta) / 2] & \text { if } a+b \text { is odd, }\end{cases} \\
y_{\ell}^{\prime}(a, b) & = \begin{cases}y_{u}^{\prime}(a-1, b+1)=[(-a+b R+1+R+\eta) / 2] & \text { if } a+b \text { is even, } \\
y_{u}^{\prime}(a, b+1)=[(-a+b R+R+\eta) / 2] & \text { if } a+b \text { is odd. }\end{cases}
\end{aligned}
$$

Note that $w^{\prime}=x_{r}^{\prime}-x_{\ell}^{\prime}$ and $h^{\prime}=y_{\ell}^{\prime}-y_{u}^{\prime}$. Every point in $R^{2}$ is in at most one block of the $P^{\prime}$ array (the proof is similar to the proof of Lemma 3.1). It follows that no blocks of $P^{\prime}$ overlap. Within each block the map is $1-1$ by definition.

Lemma 4.2. For all $r \in I_{R}, T\left(\alpha_{r}\right)$ is onto.

Proof. Every point in $R^{2}$ is in some block of the $P^{\prime}$ array (the proof is similar to the proof of Lemma 3.2). Clearly, every block of $P^{\prime}$ is in the image of $T\left(\alpha_{r}\right)$.

Theorem 4.3. For all $r \in I_{R}, e\left(T\left(\alpha_{r}\right), \alpha_{r}\right) \leq(1 / \sqrt{2})\left((1+r) / \sqrt{1+r^{2}}\right)$.

Proof. Let $r \in I_{R}$ and $z=(x, y)$. First assume that for some $a+b$ even we have

$$
\begin{aligned}
& x=x_{\ell}(a, b)+u, \\
& y=y_{u}(a, b)+v,
\end{aligned}
$$

i.e.,

$$
\begin{aligned}
& x=\left[\frac{a R-b+\zeta}{2}\right]+u, \\
& y=\left[\frac{a+b R+\eta}{2}\right]+v .
\end{aligned}
$$

We want to estimate the distance between $\left(x^{\prime}, y^{\prime}\right)$ and $\left(x^{\prime \prime}, y^{\prime \prime}\right):=\alpha_{r}(x, y)$. Recall

$$
\begin{aligned}
x^{\prime} & =x_{\ell}^{\prime}(a, b)+u, \\
y^{\prime} & =y_{u}^{\prime}(a, b)+v,
\end{aligned}
$$

i.e.,

$$
\begin{aligned}
& x^{\prime}=\left[\frac{a R+b+\zeta}{2}\right]+u, \\
& y^{\prime}=\left[\frac{-a+b R+\eta}{2}\right]+v .
\end{aligned}
$$


Also note that $\alpha_{r}$ is given by

$$
\begin{aligned}
& x^{\prime \prime}=x \cos \theta+y \sin \theta, \\
& y^{\prime \prime}=-x \sin \theta+y \cos \theta,
\end{aligned}
$$

where $\cos \theta=\left(1-r^{2}\right) /\left(1+r^{2}\right)$ and $\sin \theta=2 r /\left(1+r^{2}\right)$. The component errors in the rotation are $\Delta x=x^{\prime}-x^{\prime \prime}$ and $\Delta y=y^{\prime}-y^{\prime \prime}$. So

$$
\begin{aligned}
\Delta x= & {\left[\frac{a R+b+\zeta}{2}\right]+u-\cos \theta\left(\left[\frac{a R-b+\zeta}{2}\right]+u\right) } \\
& -\sin \theta\left(\left[\frac{a+b R+\eta}{2}\right]+v\right), \\
\Delta y= & {\left[\frac{-a+b R+\eta}{2}\right]+v+\sin \theta\left(\left[\frac{a R-b+\zeta}{2}\right]+u\right) } \\
& -\cos \theta\left(\left[\frac{a+b R+\eta}{2}\right]+v\right) .
\end{aligned}
$$

We define $\delta$ and $\gamma$ by

$$
\begin{aligned}
& {\left[\frac{a R-b+\zeta}{2}\right]=\frac{a R-b+\zeta}{2}-\delta,} \\
& {\left[\frac{a+b R+\eta}{2}\right]=\frac{a+b R+\eta}{2}-\gamma .}
\end{aligned}
$$

Note that

$$
\begin{aligned}
{\left[\frac{a R+b+\zeta}{2}\right] } & =\frac{a R+b+\zeta}{2}-\delta, \\
{\left[\frac{-a+b R+\eta}{2}\right] } & =\frac{-a+b R+\eta}{2}-\gamma .
\end{aligned}
$$

Using this and the identities

$$
\begin{aligned}
\frac{a R+b}{2} & =\cos \theta\left(\frac{a R-b}{2}\right)+\sin \theta\left(\frac{a+b R}{2}\right), \\
\frac{-a+b R}{2} & =-\sin \theta\left(\frac{a R-b}{2}\right)+\cos \theta\left(\frac{a+b R}{2}\right),
\end{aligned}
$$

we find

$$
\begin{aligned}
& \Delta x=\frac{\zeta}{2}-\delta+u-\cos \theta\left(\frac{\zeta}{2}-\delta+u\right)-\sin \theta\left(\frac{\eta}{2}-\gamma+v\right) \\
& \Delta y=\frac{\eta}{2}-\gamma+v+\sin \theta\left(\frac{\zeta}{2}-\delta+u\right)-\cos \theta\left(\frac{\eta}{2}-\gamma+v\right)
\end{aligned}
$$

or

$$
\begin{aligned}
& \Delta x=(1-\cos \theta)\left(\frac{\zeta}{2}-\delta+u\right)-\sin \theta\left(\frac{\eta}{2}-\gamma+v\right) \\
& \Delta y=\sin \theta\left(\frac{\zeta}{2}-\delta+u\right)+(1-\cos \theta)\left(\frac{\eta}{2}-\gamma+v\right)
\end{aligned}
$$


So

$$
\begin{aligned}
\Delta x^{2}+\Delta y^{2} & =\left((1-\cos \theta)^{2}+\sin ^{2} \theta\right)\left(\left(\frac{\zeta}{2}-\delta+u\right)^{2}+\left(\frac{\eta}{2}-\gamma+v\right)^{2}\right), \\
& =2(1-\cos \theta)\left(\left(\frac{\zeta}{2}-\delta+u\right)^{2}+\left(\frac{\eta}{2}-\gamma+v\right)^{2}\right) \\
& =\frac{4 r^{2}}{1+r^{2}}\left(\left(\frac{\zeta}{2}-\delta+u\right)^{2}+\left(\frac{\eta}{2}-\gamma+v\right)^{2}\right) .
\end{aligned}
$$

Now, $\zeta / 2=(3-R) / 4,0 \leq \delta<1$ and $0 \leq u \leq w-1=x_{r}-x_{\ell}-1$, so

$$
\frac{3-R}{4}-1 \leq \frac{\zeta}{2}+u-\delta \leq \frac{3-R}{4}+\frac{R+1}{2}-1 \text {, }
$$

or

$$
-\frac{1+R}{4} \leq \frac{\zeta}{2}+u-\delta \leq \frac{1+R}{4}
$$

Similarly,

$$
\left|\frac{\eta}{2}-\gamma+v\right| \leq \frac{1+R}{4}
$$

Thus we have

$$
\begin{aligned}
\Delta x^{2}+\Delta y^{2} & =\frac{4 r^{2}}{1+r^{2}}\left(2\left(\frac{R+1}{4}\right)^{2}\right), \\
& =\frac{1}{2} \frac{(1+r)^{2}}{1+r^{2}} .
\end{aligned}
$$

So

$$
\sqrt{\Delta x^{2}+\Delta y^{2}} \leq \frac{1}{\sqrt{2}} \frac{1+r}{\sqrt{1+r^{2}}} .
$$

The (easier) case where $a+b$ is odd is similar.

\section{Numerical Investigations}

\subsection{Periodicity}

Note that if $r$ is rational with $r=m / n$, then $P$ is periodic in the following sense:

$$
\begin{aligned}
& x_{\ell}(a+2 m, b)=x_{\ell}(a, b)+n, \\
& y_{u}(a+2 m, b)=y_{u}(a, b)+m, \\
& x_{\ell}(a, b+2 m)=x_{\ell}(a, b)-m, \\
& y_{u}(a, b+2 m)=y_{u}(a, b)+n .
\end{aligned}
$$

The $4 m^{2}$ blocks given, as we vary from $a$ to $a+2 m$ and from $b$ to $b+2 m$, form a fundamental domain $F$. There are $m^{2}+n^{2}$ "pixels" in each fundamental domain. When computationally studying the rational case, it suffices to restrict consideration to a fundamental domain. 


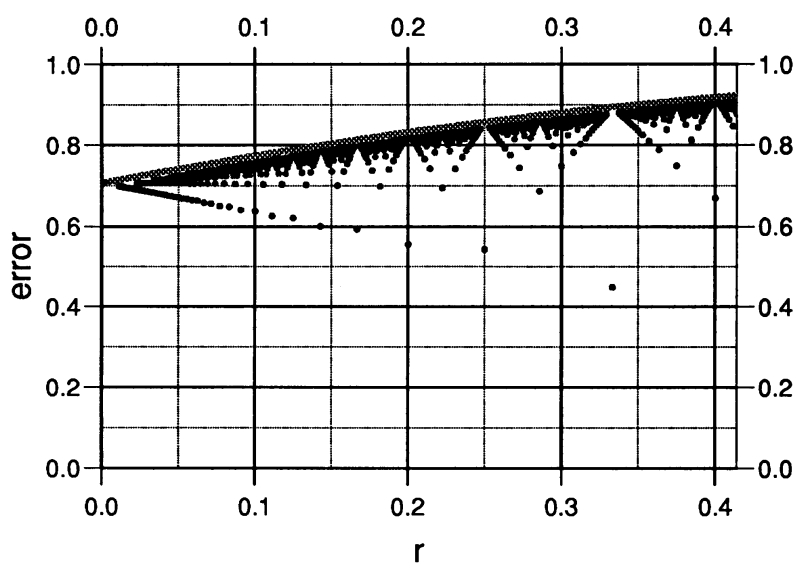

Fig. 3. Modified T-Scheme.

\subsection{Improvements}

The T-Scheme described in this paper was chosen for ease of exposition and is not optimal. It can happen that the choice

$$
\zeta=\zeta^{\prime}=\eta=\eta^{\prime}=\frac{3-R}{2}
$$

is not the best one. In particular, sometimes the choice

$$
\zeta=\frac{5-R}{2}, \quad \zeta^{\prime}=\frac{1-R}{2}, \quad \eta=\frac{3-3 R}{2}, \quad \eta^{\prime}=\frac{3-3 R}{2}
$$

is better (and sometimes it is worse). We define the Modified T-Scheme by choosing the better of these two choices (we suspect there is never a better choice). We do not make any theoretical claims concerning this improvement. (Theorem 4.3 is unchanged.) In Fig. 3 we show the error bound given by Theorem 4.3 and also the actual errors (using the Modified T-Scheme) for a sample of angles.

\subsection{Lower Bounds for "Rational” Angles}

Proposition 5.1. For all $S, E_{S}>0.787$. In fact, for all $\beta, e\left(\beta, \alpha_{\sqrt{2}-1}\right)>0.787$.

Proof. Assume not. Say $\beta$ approximates rotation by $\pi / 4$ with error less than 0.787 . $\beta$ must send the point $(0,0)$ to $(0,0)$. The point $(1,0)$ gets sent into some square and $\beta$ must send it to a corner of this square which (i) is not already in the image of $\beta$ and (ii) is within a distance 0.787 of the image point. Now the point $(1,1)$ likewise, etc. This forms a simple tree of possibilities which can be checked until a contradiction is obtained. We did this for some selected angles (see Fig. 4). The maximum we found (via a deeper search than shown in Fig. 4) was 0.787 at " $\pi / 4$." 


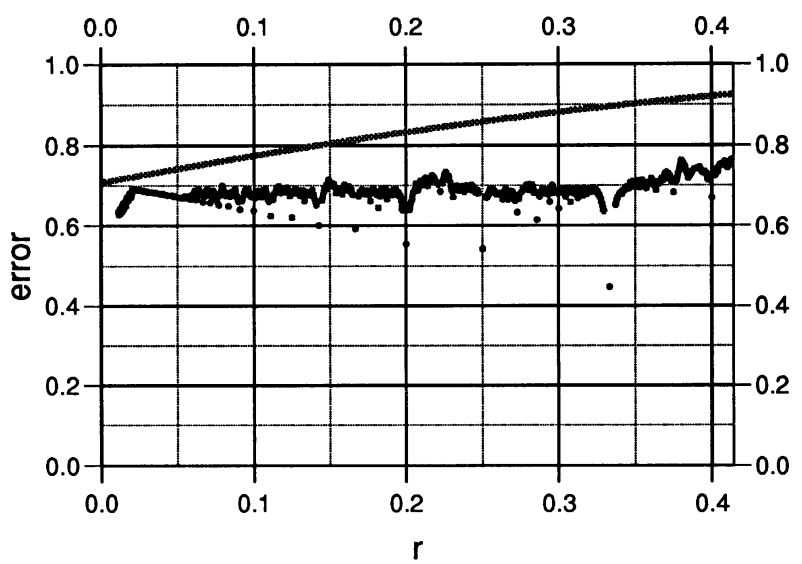

Fig. 4. Lower bounds for "rational" angles.

Remark 5.2. Note that the graphs indicate that the Modified T-Scheme is not optimal. The program actually constructs candidates for better $\beta$ 's; we hope to investigate these in the future. In the simplest case, $\frac{2}{7}$, we have found the computer's candidate and have checked that it is indeed better.

Remark 5.3. To emphasize the simplicity of the program, we give the code (without declarations):

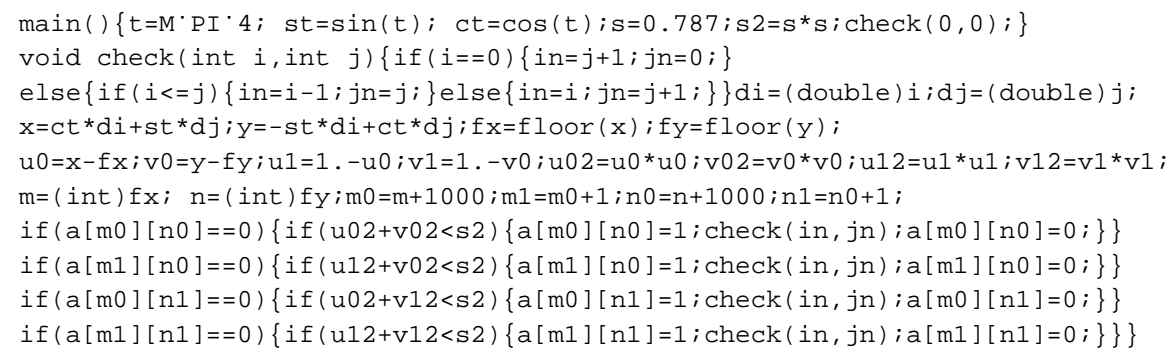

\subsection{Lower Bounds for "Irrational" Angles}

As indicated by Fig. 4 and by experience, the "worst" case seems to occur at $\pi / 4$.

Example 5.4. (Due to Richard Stong.) Suppose we are trying to approximate a rotation $x \rightarrow a(x)$ by an angle of $\pi / 4$ by a bijection. There will be some point $x=(n, 0)$ such that $a(x)=(n / \sqrt{2}, n / \sqrt{2})$ is very close to $(m+0.5, m+0.5)$. Therefore, the images of the four points $(n,-1),(n, 1),(n-1,0)$, and $(n+1,0)$ will be approximately at $(m+0.5+\sqrt{2} / 2, m+0.5+\sqrt{2} / 2),(m+0.5-\sqrt{2} / 2, m+0.5+\sqrt{2} / 2),(m+0.5-$ $\sqrt{2} / 2, m+0.5-\sqrt{2} / 2)$, and $(m+0.5+\sqrt{2} / 2, m+0.5-\sqrt{2} / 2)$. The images of these 
five points will all be reasonably close to the four lattice points which are vertices of the unit square containing $(m+0.5, m+0.5)$. However, the approximating bijection must pair one of these five points to a point not on that square. Up to symmetry it does not matter which, and we see that one of the five images must be moved a distance $D$ which approximately satisfies

$$
D^{2}=\frac{7-4 \sqrt{2}}{2}
$$

Hence $D$ is about 0.819.

By generalizing this example we have

Theorem 5.5. For all $S, E_{S} \geq(2+\sqrt{2}) / 4 \approx 0.853$. In fact, for all $\beta, e\left(\beta, \alpha_{\sqrt{2}-1}\right) \geq$ $(2+\sqrt{2}) / 4$.

Proof. Continue using the previous example and consider all the image points which lie within the square with corners given by the image points $(m+0.5+e(\sqrt{2} / 2), m+0.5+$ $e(\sqrt{2} / 2)),(m+0.5-e(\sqrt{2} / 2), m+0.5+e(\sqrt{2} / 2)),(m+0.5-e(\sqrt{2} / 2), m+0.5-$ $e(\sqrt{2} / 2))$, and $(m+0.5+e(\sqrt{2} / 2), m+0.5-e(\sqrt{2} / 2))$. The example is the case where $e=1$. There are $b_{e}=2 e^{2}+2 e+1$ such image points. Let $\varepsilon_{e}=((\sqrt{2} / 2)+e) \bmod \sqrt{2}$, $s_{e}=\sqrt{2}\left(e-\varepsilon_{e}\right)$, and $r_{e}=1-(\sqrt{2} / 2) \varepsilon_{e}$. Clearly the $b_{e}$ open balls of radius $r_{e}$ around the image points cover at most $c_{e}=\left(s_{e}+1\right)^{2}$ pixels. We consider only the cases where $b_{e}-c_{e}=1$. A simple calculation shows that this occurs if and only if $(\sqrt{2} / 2)+e-\varepsilon_{e}$ is an integer multiple of $\sqrt{2}$ and $\varepsilon_{e}=\sqrt{2} / 2+e-\sqrt{e^{2}+e}$.

To prove that infinitely many $e$ 's exist with $b_{e}-c_{e}=1$ we use Theon of Smyrna's (AD 130) sequence of ratios approximating $\sqrt{2}$, called "side-and-diagonal numbers":

$$
d_{1} / s_{1}=1 / 1,3 / 2,7 / 5,17 / 12, \ldots, d_{n} / s_{n}, \ldots
$$

with $s_{n+1}=s_{n}+d_{n}$ and $d_{n+1}=2 s_{n}+d_{n}$. In particular for all $n, s_{2 n}$ is even and $d_{2 n}^{2}=1+2 s_{2 n}^{2}$. Now let $e_{n}=\left(d_{2 n}-1\right) / 2$ and let $\varepsilon_{n}=\sqrt{2} / 2+e_{n}-\sqrt{e_{n}^{2}+e_{n}}$. A short calculation shows that $\sqrt{2} / 2+e_{n}-\varepsilon_{n}$ is an integer multiple of $\sqrt{2}$.

Note that $\lim _{n \rightarrow \infty} r_{n}=(2+\sqrt{2}) / 4$ as required.

For "irrational" angles some pixel is sent infinitesimally close to the center of a square. Modifying the computer program of Section 5.3 accordingly, we obtain Fig. 5 which shows lower bounds for irrationals infinitesimally close to the rationals calculated by the computer.

\section{Open Problems}

- What is $M:=\inf _{S}\left\{E_{S}\right\}$ where $S$ ranges over all schemes? (We have proven that $0.853<(2+\sqrt{2}) / 4 \leq M \leq \sqrt{(2+\sqrt{2}) / 4}<0.924$. $)$

- Does there exist a scheme (or set of schemes) $S^{*}$ with $E_{S^{*}}=M$ ? Can it (or they) be described explicitly? 


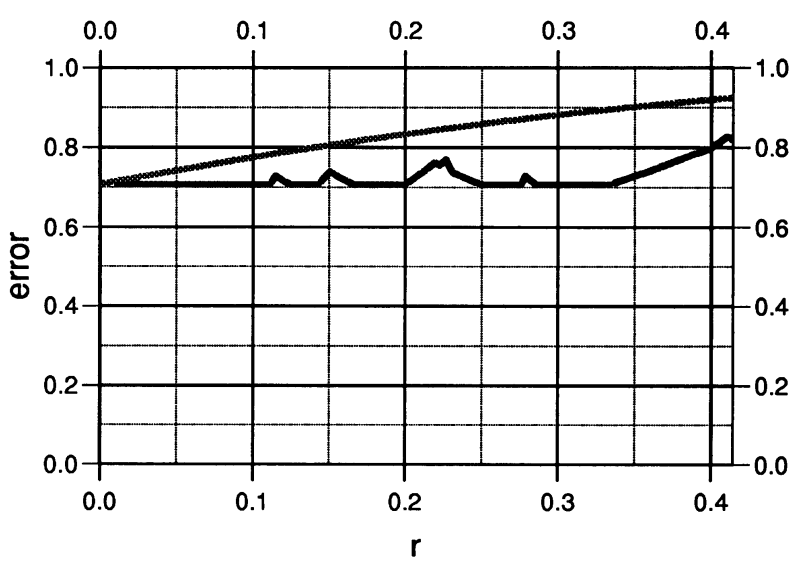

Fig. 5. Lower bounds for "irrational" angles.

- More generally, what is the function $M(\alpha):=\inf _{\beta \in L}\{e(\beta, \alpha)\}$ ? (We have proven that Lower $\left(\alpha_{r}\right)<M\left(\alpha_{r}\right) \leq(1 / \sqrt{2})\left((1+r) / \sqrt{1+r^{2}}\right)$, where Lower is the lower graph in Fig. 4 (Fig. 5 for "irrationals"), which we have only checked for some selected angles.)

- What happens if the approximations are not required to be 1-1? Onto? If approximations are not required to be 1-1 nor onto, then clearly the "nearest neighbor" scheme is theoretically optimal, although it may be cpu intensive.

- What happens if the goodness of a scheme is measured by how well it does "on the average" as opposed to "in the worst case" as we have here?

- Richard Stong observed, using Hall's Marriage Theorem, that a scheme that works is obtained unless a configuration as above, where the rotates of $k$ points are within $r$ of only a total of $k-1$ points, is found. This condition certainly seems to simplify the problem, but we cannot yet prove which "clusters" are optimal nor which angles are "worst."

\section{Reference}

[T] Sterling, T., Image Rotation Using Block Transfers, U.S. Pat. No. 5,359,706, Oct. 25, 1994.

Received November 21, 1996, and in revised form February 20, 1997. 